\title{
A Novel Fluorescent Probe for Copper Ions Based on Polymer-modified CdSe/CdS Core/Shell Quantum Dots
}

\author{
Zhong CaO, ${ }^{*}, *+$ Zheng Gu,** Ju-Lan Zeng, ${ }^{*}$ Jin-Hua LiU, ${ }^{* *}$ Qin Deng, ${ }^{* *}$ Jun-Bing FAn, ${ }^{* *}$ and \\ Jian-Nan XIANG ${ }^{* * \dagger}$
}

*Hunan Provincial Key Laboratory of Materials Protection for Electric Power and Transportation, School of Chemistry and Biological Engineering, Changsha University of Science and Technology, Changsha 410004, P. R. China

**State Key Laboratory of Chemo/Biosensing and Chemometrics, College of Chemistry and Chemical Engineering, Hunan University, Changsha 410082, P. R. China

\begin{abstract}
Quantum dots (QDs) have become one of the most attractive fields of current research because of their unique optical properties. Novel copper-sensitive fluorescent fluoroionophores based on $\mathrm{CdSe} / \mathrm{CdS}$ core/shell QDs modified with a polymer of MAO-mPEG were synthesized and characterized in the present work. A pH of 6.47 was optimally selected for measurements. By modifying QDs with MAO-mPEG, significant aqueous fluorescence quenching was observed upon binding with copper ions involving both reduced and oxidized environments, indicating great sensitivity and specificity for copper-ion sensing. No significant interfering effects from other metal ions, such as $\mathrm{Ag}^{+}, \mathrm{Al}^{3+}, \mathrm{Ba}^{2+}, \mathrm{Ca}^{2+}, \mathrm{Cd}^{2+}, \mathrm{Co}^{2+}$, $\mathrm{Cr}^{3+}, \mathrm{Fe}^{2+}, \mathrm{Fe}^{3+}, \mathrm{Hg}^{2+}, \mathrm{K}^{+}, \mathrm{Mg}^{2+}, \mathrm{Mn}^{2+}, \mathrm{Na}^{+}, \mathrm{Ni}^{2+}, \mathrm{Pb}^{2+}, \mathrm{Sn}^{2+}$, and $\mathrm{Zn}^{2+}$, were observed. The linear response range for $\mathrm{Cu}^{2+}$ was found to be $0.01-0.50 \mu \mathrm{M}$, and the limit of detection was evaluated to be $16 \mathrm{nM}$. The proposed method demonstrated improved sensitivity and selectivity characteristics for $\mathrm{Cu}$ (II) determinations based on $\mathrm{CdSe} / \mathrm{CdS}$ core/shell QDs modified with MAO-mPEG by using a typical liquid-phase quenching assay, showing its potential application to multiplex sensing of different analytes through distinct ligand conjugation and functionalization of individual fluorophores.
\end{abstract}

(Received November 23, 2010; Accepted March 24, 2011; Published June 10, 2011)

\section{Introduction}

Photoluminescent semiconductor nanocrystals or quantum dots (QDs) were of great fundamental scientific interest and industrial applications due to their size-dependent photoluminescent properties. ${ }^{1-5}$ QDs have the property that upon radiation energy can be absorbed (at any wavelength greater than the energy of their lowest energy transition) and converted into narrow bandwidth emission close to the band edge. ${ }^{6}$ Compared with organic fluorescent dyes, the QDs exhibited some important advantages, including large fluorescence quantum yields, narrow and Gaussian emission spectra (full height wide maximum of $30 \mathrm{~nm}$ ), tunable maximum wavelength of emission by controlling the QD size (varying the size from 1 to $7 \mathrm{~nm}$ allows most visible colors to be generated) and less susceptibility to photobleaching. ${ }^{7,8}$ Until now, QDs were valuable for many applications ranging from single-electron transistors to biomarkers. ${ }^{9}$

Surface-modified QDs may change their optical, chemical and photocatalytic properties and can give rise to such effects as an enhancement of their excitonic and defect emission, an improvement of the photostability of semiconductor nanoparticles, the generation of new traps on the surface of the $\mathrm{QD}$, leading to the appearance of new emission bands, an

$\dagger$ To whom correspondence should be addressed. E-mail: zhongcao04@yahoo.com.cn;jnxiang@hnu.cn enhancements of the selectivity and efficiency of light-induced reactions occurring on the surface of semiconductor nanoparticles, etc. ${ }^{10}$

For routine preparation, the CdSe core was usually capped with an organic layer, such as a trioctylphosphine/trioctylphosphine oxide mixture (TOP/TOPO). This layer is coordinated to $\mathrm{Cd}$ sites and stabilizes QDs' surface, while preventing an irreversible flocculation of the nanocrystals. ${ }^{11}$ Unfortunately, these protective ligands were hydrophobic, and thus nanocrystals capped with those coatings were not compatible with analytically useful aqueous assay conditions. Consequently, the QDs surface should be further modified by appropriate hydrophilic functional groups to allow their dispersion in aqueous solutions while maintaining their high photoluminescence quantum yield. ${ }^{12}$ In this way, the tailoring of nanoparticles for different purposes could be achieved.

Notwithstanding the great potential of luminescent QDs in chemical analysis, their analytical applications in aqueous media were still scarce, although this was rapidly changing as several papers have very recently appeared in this field. For example, the use of modified CdSe QDs for the sensitive determination of cyanide ions has been proposed. ${ }^{13}$ Also, water-soluble CdS QDs whose luminescences were quenched by copper and zinc $^{14}$ or copper and silver ${ }^{15}$ have been investigated. CdSe/ZnS core/shell QDs modified with bovine serum albumin were also investigated for the determination of copper, ${ }^{16}$ while CdSe QDs modified with mercaptoacetic acid and bovine serum albumin were assayed for the analysis of silver. ${ }^{17}$ In addition, CdTe 


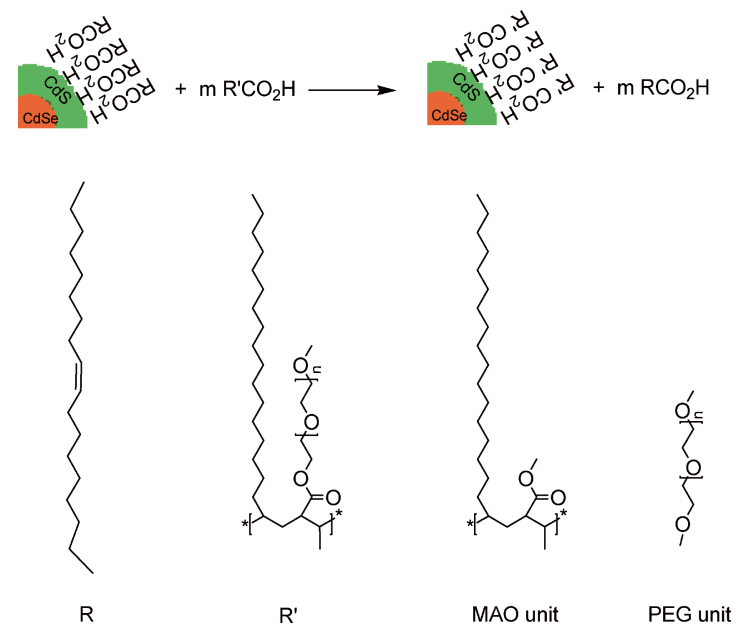

Fig. 1 Synthesis process of QDs-MAO-mPEG.

nanocrystals modified with mercaptopropionic acid were proposed for the determination of $\mathrm{Cu}$ (II) ions. ${ }^{18}$ A lack of selectivity and low stability of the QDs in the aqueous media were among the problems reported for some of the procedures proposed.

In the present work, the selective determination of $\mathrm{Cu}$ (II) in aqueous solutions based on CdSe/CdS core/shell QDs modified either with oleic acid or MAO-mPEG was thoroughly investigated, showing its promising application commenting the luminescent spectrometric analysis of metal ions.

\section{Experimental}

\section{Reagents}

The following polymer materials were used: poly(maleic anhydride-alt-1-octadecene) $\quad\left(\mathrm{MAO}, \quad M_{\mathrm{n}}=30000-50000\right.$, $M_{\mathrm{w}}=350.5$, Aldrich, St. Louis, MO); poly(ethylene glycol) methyl ethers (mPEG-OH, $M_{\mathrm{w}}=750$, Aldrich, USA). Other chemicals were of analytical reagent grade, which were purchased from a local chemicals company. All used solvents were distilled prior to use.

\section{Prepare of water-soluble CdSe/CdS core/shell QDs}

CdSe QDs were synthesized using $\mathrm{CdO}$ as a precursor according to a procedure described by Peng's group; ${ }^{3,19}$ the $\mathrm{CdSe} / \mathrm{CdS}$ core/shell QDs were prepared in trioctylphosphine oxide (TOPO) as a solvent.

For the synthesis of MAO-mPEG, MAO (4.002 g, $0.10 \mathrm{mmol})$ reacted with mPEG-OH $(6.043 \mathrm{~g}, 8.02 \mathrm{mmol})$ to make MAO-mPEG amphiphilic polymers, under acid catalysis (e.g., two drops of concentrated $\left.\mathrm{H}_{2} \mathrm{SO}_{4}\right)$ in chloroform $(10 \mathrm{~mL})$ and reflux at $70^{\circ} \mathrm{C}$ for $12 \mathrm{~h}$. The solution was neutralized by $1 \mathrm{~mol} / \mathrm{L} \mathrm{NaOH}$, to which distilled water $(30 \mathrm{~mL})$ was added, extracted by chloroform $(15 \mathrm{~mL})$, and dried; a yellow solid (yield was $85 \%$ ) was obtained.

For the synthesis of QDs-MAO-mPEG in Fig. 1, CdSe/CdS core/shell QDs $(0.1 \mathrm{~mL}, \sim 0.5 \mathrm{nmol})$, oleamine $(0.08 \mathrm{~mL}$, $0.24 \mathrm{mmol}$ ) and $1 \mathrm{~mL}$ acetone (already dry processing) were added in a plastic tube, then centrifuged and dissolved with a 1-mL THF solution of MAO-mPEG (30 mg, $27 \mathrm{nmol}$ ). After the solvent was evaporated, the residue was dissolved with a $\mathrm{NaOH}$ solution ( $\mathrm{pH}$ 9). The nanocrystals dissolved completely, and the solution was filtered to remove away excess unbound

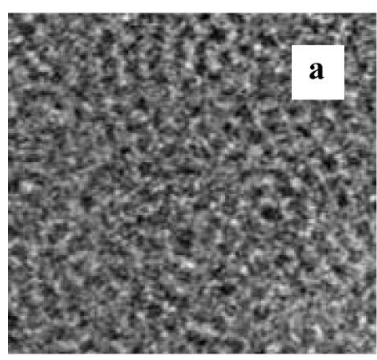

$10 \mathrm{~nm}$

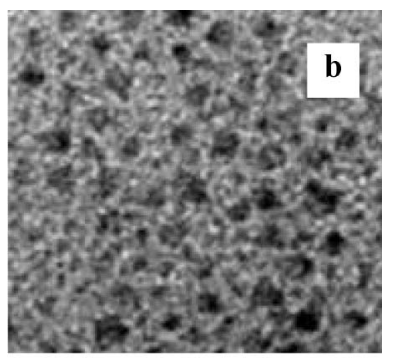

$10 \mathrm{~nm}$
Fig. 2 TEM images of (a) original CdSe/CdS core/shell QDs and (b) $\mathrm{CdSe} / \mathrm{CdS}$ core/shell QDs capped with MAO-mPEG; scale bars are both $10 \mathrm{~nm}$.

polymer. The buffer was exchanged with water by two rounds of dilution and reconcentration through a centrifuge filter. Any residual unbound polymer was removed by two consecutive purification steps on a size-exclusion column. After purification, the nanocrystal solutions were optically clear.

\section{Results and Discussion}

\section{Self-assembled giant MAO vesicles}

Self-reproductive micelles and vesicles have been the focus of intensive investigations for several decades because the motivation for this work originated in a definition of the minimal conditions necessary for a system. Fatty acid vesicles are colloidal suspensions of closed lipid bilayers that are composed of fatty acids and their ionized species (soap). Compared with diacylglycerophospholipid vesicles (conventional liposomes), one important feature of fatty acid vesicles is that dynamic nature owing to the fact that they are composed of single chain amphiphiles. The concentration of non-associated monomers in equilibrium with vesicles is considerably higher than in the case of double-chain phospholipids. In addition, a range of fatty acid self-assembled nanostructures are formed just by changing the total concentration and the protonation/ionization ratio of the terminal carboxylic acid. Further exploration on fatty acid vesicles as protocell models or fatty acid-based vesicles as drug delivery or food additive systems is still necessary.

As similar molecules to oleic acid in a polymer, the hydrolyzed derivative of MAO could be regarded as being polymerized oleic acid. The only main difference is that each MAO unit contains two hydrocarboxy groups, while having oleic acid with only one hydrocarboxy group. As described above, the vesicles assembled from fatty acids and their ionized species (soap) are much more flexible than conventional liposomes. However, MAO self-assembled vesicles would be more stable than oleic acid and liposomes because MAO unit contains many chains, while there are one (oleic acid) or two chains (liposomes) with each building block. Therefore, it is possible to observe a self-assembly of MAO under different solution conditions.

The particles were characterized by transmission electron microscopy (TEM, JEOL-JEM 2010, JEOL Co. Ltd., Japan), which was operated at $200 \mathrm{kV}$. The solutions were stable for months (i.e., no precipitation occurred and the bands during gel electrophoresis kept their narrow shape). As shown in TEM images (Figs. 2a and 2b), the diameters of the QD particles after surface modification increased from $1.6 \pm 0.7 \mathrm{~nm}$ to $3.7 \pm 1.1 \mathrm{~nm}$ and the QDs were monodisperse and uniform. Therefore, the observed increase in particle size by TEM images 


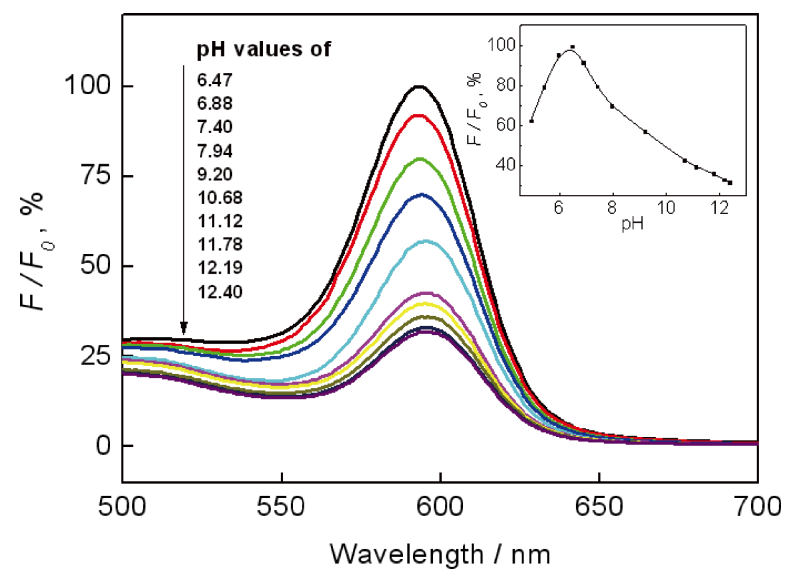

Fig. 3 Effect of the pH on the fluorescence of QDs-MAO-mPEG $(1.0 \mu \mathrm{M}, 298 \mathrm{~K}$, potassium dihydrogen phosphate and disodium phosphate ( $\mathrm{v}: \mathrm{v}=1: 1)$ buffer solution, $\lambda_{\mathrm{ex}}=400 \mathrm{~nm}$ ). The $\mathrm{pH}$ value was adjusted by $\mathrm{HClO}_{4}$ and tetramethylammonium hydroxide.

can be attributed to a polymer-layer coated on the surface of QDs.

\section{Investigation on interference}

The interference from other ions in aqueous solution is a very important factor to be considered for a fluoroionophore in real sample measurements. The fluorescent detection of $\mathrm{Cu}^{2+}$ in potassium dihydrogen phosphate and disodium phosphate (v:v = 1:1) buffer solution was generally influenced, more or less, by interference from some metal ions. The effect of the $\mathrm{pH}$ on the fluorescence of QDs-MAO-mPEG was first determined by fluorescence titration in the buffer solution, which is shown in Fig. 3. It can be seen that the fluorescence of QDs-MAO-mPEG at $595 \mathrm{~nm}$ remains affected at between $\mathrm{pH} 12.40$ and 4.92, and the optimal $\mathrm{pH}$ of $6.47 \mathrm{can}$ be selected for further fluorescence studies.

The competence of QDs-MAO-mPEG in detecting various metal ions has been investigated in detail. Figure 4 illustrates the spectroscopic response of the QDs-MAO-mPEG sensor following the addition of a variety of miscellaneous metal ions (including $\mathrm{Ag}^{+}, \mathrm{Al}^{3+}, \mathrm{Ba}^{2+}, \mathrm{Ca}^{2+}, \mathrm{Cd}^{2+}, \mathrm{Co}^{2+}, \mathrm{Cr}^{3+}, \mathrm{Cu}^{+}, \mathrm{Cu}^{2+}$, $\left.\mathrm{Fe}^{2+}, \mathrm{Fe}^{3+}, \mathrm{Hg}^{2+}, \mathrm{K}^{+}, \mathrm{Mg}^{2+}, \mathrm{Mn}^{2+}, \mathrm{Na}^{+}, \mathrm{Ni}^{2+}, \mathrm{Pb}^{2+}, \mathrm{Sn}^{2+}, \mathrm{Zn}^{2+}\right){ }^{20}$ It can be seen that the fluorescence of the QDs-MAO-mPEG sensor is essentially unaffected by the presence of these common ions, except for $\mathrm{Cu}^{2+}$ and $\mathrm{Cu}^{+}$, while the metal-ion concentration is lower than $500 \mathrm{nM}$. Therefore, under concentrations of $\mathrm{Cu}^{2+}$ and $\mathrm{Cu}^{+}$ions lower than $500 \mathrm{nM}$, the polymer-coated QDs exhibits an excellent selectivity toward $\mathrm{Cu}^{+}, \mathrm{Cu}^{2+}$ above other interfering metal ions. Interestingly, it has been demonstrated that QDs-MAO-mPEG could be simultaneously utilized in the detection of both $\mathrm{Cu}^{2+}$ and $\mathrm{Cu}^{+}$involving an oxidized and reduced environment, on which few reports have been focused. Further investigations on various metal ions with different concentrations have been performed; Fig. 5a shows the relative fluorescence intensity of QDs-MAO-mPEG $(1.0 \mu \mathrm{M})$ at $400 \mathrm{~nm}$ excitation with the titrations of $\mathrm{Pb}^{2+}, \mathrm{Mn}^{2+}, \mathrm{Zn}^{2+}, \mathrm{Sn}^{2+}, \mathrm{Cd}^{2+}$, $\mathrm{Mg}^{2+}, \mathrm{Ag}^{+}, \mathrm{Hg}^{2+}, \mathrm{Cu}^{+}$, and $\mathrm{Cu}^{2+}$ at different concentrations (pH 6.47). It can be seen that the QDs-MAO-mPEG show better selectivity when the metal-ion concentraion is lower than $1.0 \mu \mathrm{M}$, which possesses great sub-micromolar scale sensitivity. Also, a comparison experiment was carried out with QDs-MAO-mPEG in the presence of metal ions other than copper ions. Figure $5 \mathrm{~b}$ shows the relative fluorescence intensity

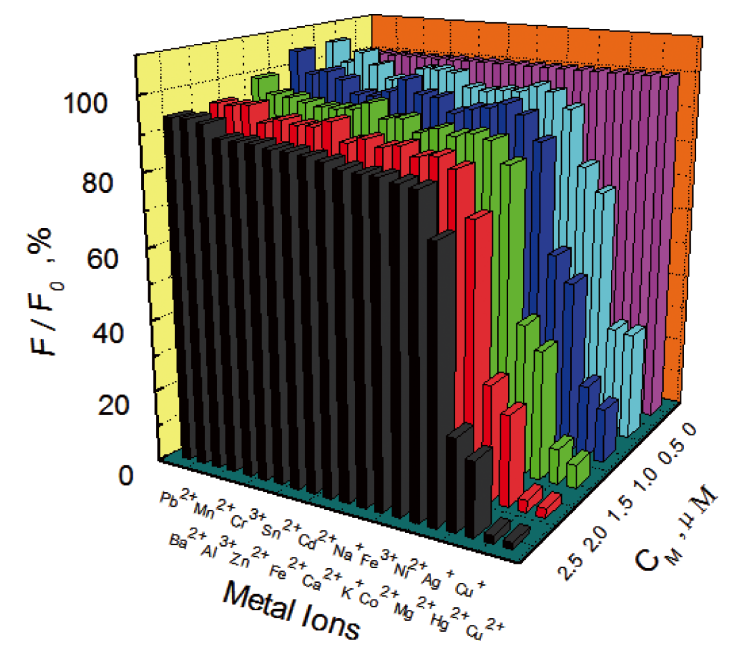

Fig. 4 Relative fluorescence intensity at $595 \mathrm{~nm}$ for QDs-MAO-mPEG $(1.0 \mu \mathrm{M}, 298 \mathrm{~K}$, potassium dihydrogen phosphate and disodium phosphate $(\mathrm{v}: \mathrm{v}=1: 1)$ buffer solution) at $400 \mathrm{~nm}$ excitation, and after adding different metal ions $(0-2.5 \mu \mathrm{M})$ for $2 \mathrm{~min}$.
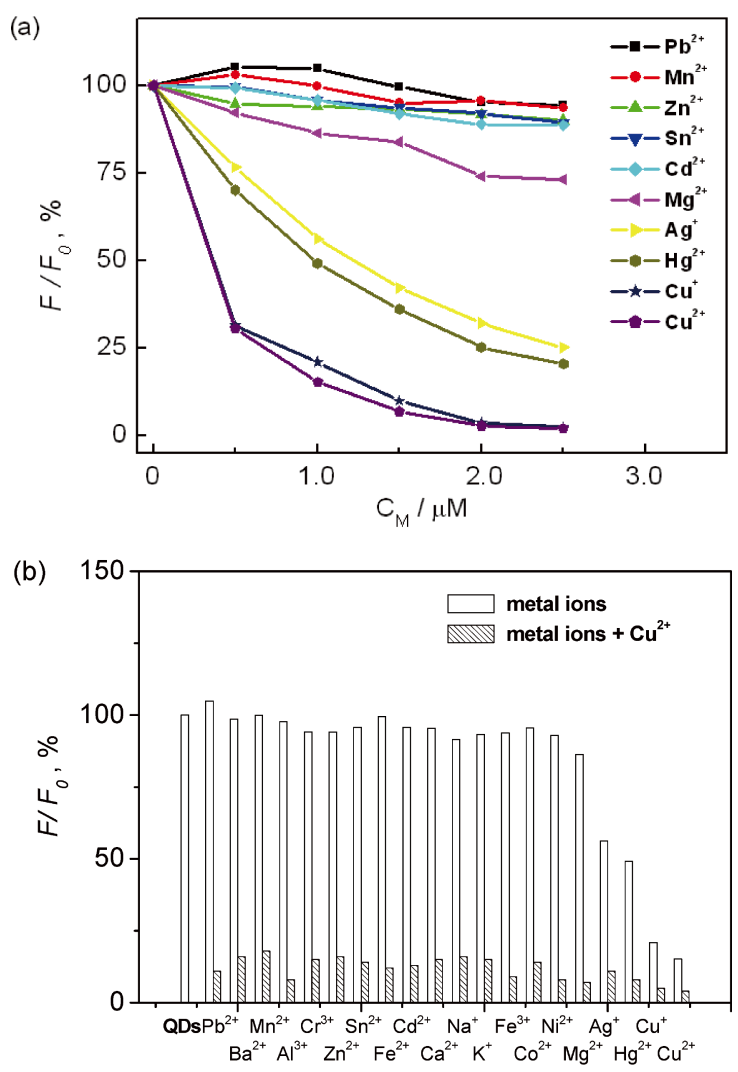

Fig. 5 (a) Relative fluorescence intensity of QDs-MAO-mPEG $(1.0 \mu \mathrm{M}, 298 \mathrm{~K}$, potassium dihydrogen phosphate and disodium phosphate (v:v = 1:1) buffer solution) at $400 \mathrm{~nm}$ excitation with the titrations of $\mathrm{Pb}^{2+}, \mathrm{Mn}^{2+}, \mathrm{Zn}^{2+}, \mathrm{Sn}^{2+}, \mathrm{Cd}^{2+}, \mathrm{Mg}^{2+}, \mathrm{Ag}^{+}, \mathrm{Hg}^{2+}, \mathrm{Cu}^{+}$, and $\mathrm{Cu}^{2+}$ at different concentrations ( $\mathrm{pH}$ 6.47). (b) Relative fluorescence intensity of QDs-MAO-mPEG $(1.0 \mu \mathrm{M}, 298 \mathrm{~K})$ in the presence of various metal ions alone $(1.0 \mu \mathrm{M})$ and interfering ions with $\mathrm{Cu}^{2+}$ $(1.0 \mu \mathrm{M})$, respectively. $\lambda_{\mathrm{ex}}=400 \mathrm{~nm}, \lambda_{\mathrm{em}}=595 \mathrm{~nm}$.

of QDs-MAO-mPEG $(1.0 \mu \mathrm{M})$ in the presence of various metal ions alone $(1.0 \mu \mathrm{M})$ and interfering ions with $\mathrm{Cu}^{2+}(1.0 \mu \mathrm{M})$, 
respectively. It can be seen that the addition of $\mathrm{Cu}^{2+}(1.0 \mu \mathrm{M})$ to a QDs-MAO-mPEG solution containing any one of the above-mentioned metal ions $(1.0 \mu \mathrm{M})$ results in a similar amount of quenching of $80-95 \%$ on the fluorescence intensity. Thus, the presence of these metal ions with $\mathrm{Cu}^{2+}$ has no significant interfering effect on the sensitivity of QDs-MAO-mPEG for $\mathrm{Cu}^{2+}$ detection.

\section{Sensitivity of QDs-MAO-mPEG}

The sensitivity of QDs-MAO-mPEG for $\mathrm{Cu}^{2+}$ detection on fluorescence quenching has been investigated in detail. The maximum excitation and emission wavelengths were obtained at 400 and $595 \mathrm{~nm}$ from the fluorescence spectra of QDs-MAO-mPEG, respectively. Figure 6a shows fluorescence emission spectra of QDs-MAO-mPEG $(1.0 \mu \mathrm{M})$ in the presence of an increasing concentration of $\mathrm{Cu}^{2+}$. The fluorescence intensity of $34 \%$ was quenched when $0.1 \mu \mathrm{M}$ of $\mathrm{Cu}^{2+}$ was added (Fig. 6a inset). Figure $6 \mathrm{~b}$ shows the relative fluorescence intensity of QDs-MAO-mPEG with increasing $\mathrm{Cu}^{2+}$ concentration. It can be seen that the emission intensity of QDs-MAO-mPEG decreases abruptly with increasing $\mathrm{Cu}^{2+}$ concentration, and finally reachs a plateau at about $1.5 \mu \mathrm{M}$, which indicates a hyperbolic curve. The experimental curve of fluorescence quenching was obtained by a reciprocal treatment (Fig. 6b inset). Fluorescence quenching can be described by the Stern-Volmer equation,

$$
\frac{F_{0}}{F}=1+k_{\mathrm{q}} \tau_{0}[\mathrm{Q}]=1+K_{\mathrm{SV}}[\mathrm{Q}]
$$

where $F_{0}$ and $F$ are the fluorescence intensities before and after the addition of the quencher, respectively; $k_{\mathrm{q}}$ is the bimolecular quenching constant; $\tau_{0}$ the lifetime of the fluorophore in the absence of quencher; [Q] the concentration of the quencher, and $K_{\mathrm{SV}}$ the Stern-Volmer quenching constant. Accordingly, the relationship between $F_{0} / F$ and the $\mathrm{Cu}^{2+}$ concentration was obtained as $F_{0} / F=4.443 \times 10^{6}\left[\mathrm{Cu}^{2+}\right]+1$. The linear range of QDs-MAO-mPEG for $\mathrm{Cu}^{2+}$ detection was then found to be $0.01-0.50 \mu \mathrm{M}$ with a correlation coefficient of $R_{2}=0.99872$. The Stern-Volmer quenching constant was estimated to be $4.443 \times 10^{6} \mathrm{M}^{-1}$. The detection limit of the QDs-MAO-mPEG for $\mathrm{Cu}^{2+}$ detection was found to be $16 \mathrm{nM}$ from 9 blank solutions according to the IUPAC definition $\left(C_{\mathrm{DL}}=3 S_{\mathrm{b}} / m\right){ }^{21}$ Thus, the formation constant of QDs-MAO-mPEG with $\mathrm{Cu}^{2+}$ could be calculated as $2.88 \times 10^{6} \mathrm{M}^{-1}$ according to a method reported elsewhere. ${ }^{22}$

Compared with the reported QDs analytical systems, ${ }^{13-18}$ the difference is that our presented QDs is based on the CdSe/CdS core/shell and the QDs surface is modified by an amphiphilic polymer of MAO-mPEG. Until now, there were not very clear mechanisms for the interpretation of fluorescent quenching of QDs with heavy metal ions. ${ }^{23}$ Several quenching mechanisms including inner filter effects, nonradiative recombination pathways, electron-transfer processes, and ion binding interactions have been proposed to explain how metal ions quench the fluorescence of QDs..$^{24,25}$ A possible mechanism of our detected system could be understood from the fitted Stern-Volmer relationship, which describes the concentration of the $\mathrm{Cu}$ (II) ion dependence on the luminescence intensity of QDs-MAO-mPEG. As seen from an inert plot of Fig. 6b, a very good linearity of $F_{0} / F v s$. $\left[\mathrm{Cu}^{2+}\right]$ is observed, which is in consistent agreement with the Stern-Volmer Eq. (1). Because the QDs surface was modified by the amphiphilic polymer of MAO-mPEG with hydrophilic hydrocarboxy groups, it could allow for nanocrystals well dispersed in aqueous solution while (a)

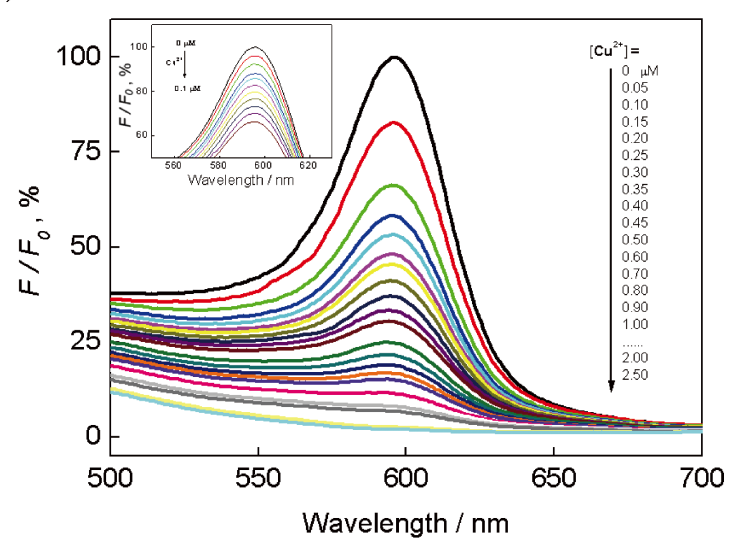

(b)

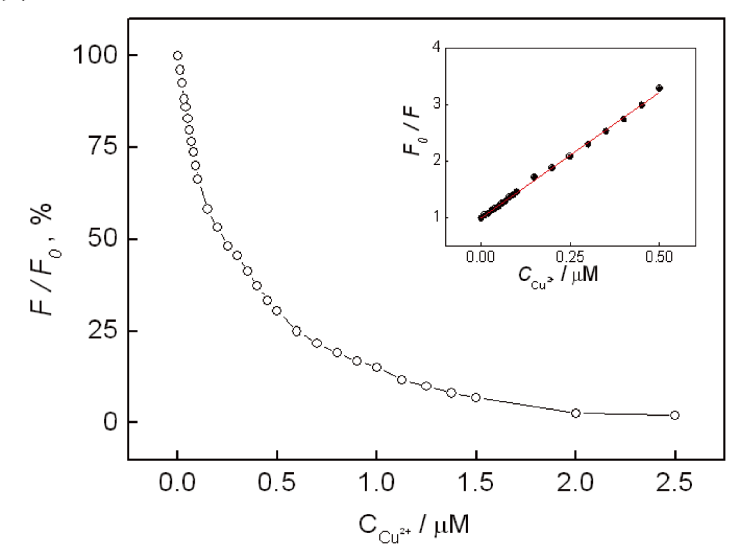

Fig. 6 (a) Fluorescence emission spectra of QDs-MAO-mPEG $(1.0 \mu \mathrm{M}, 298 \mathrm{~K}$, potassium dihydrogen phosphate and disodium phosphate ( $\mathrm{v}: \mathrm{v}=1: 1)$ buffer solution) in the presence of increasing concentration of $\mathrm{Cu}^{2+}$. The concentrations of $\mathrm{Cu}^{2+}$ for the bottom to top curves are $0,0.05,0.10,0.15,0.20,0.25,0.30 \ldots 2.50 \mu \mathrm{M}$. Inset: fluorescence emission spectra of $1.0 \mu \mathrm{M}$ QDs-MAO-mPEG in the presence of $0-0.1 \mu \mathrm{M} \mathrm{Cu}^{2+}$. (b) Relative fluorescence intensity of QDs-MAO-mPEG with increasing $\mathrm{Cu}^{2+}$ concentration. Fluorescence intensity at $\lambda_{\mathrm{em}}=595 \mathrm{~nm}$ as a function of the copper concentration. Inset: $F_{0} / F$ at $595 \mathrm{~nm}$ as a function of the $\mathrm{Cu}^{2+}$ concentration within $0-0.50 \mu \mathrm{M}$ range. Excitation wavelength, $400 \mathrm{~nm}$.

maintaining their high photoluminescence quantum yield. Compared with other metal ions, the copper ion could easily go through the mobile pathway/cavity of the polymer of MAO-mPEG on the surface of QDs by its appropriate radium size, and reached the surface to form $\mathrm{Cu}_{\mathrm{x}} \mathrm{S}(x=1,2)$ by substituting some $\mathrm{Cd}$ atoms of $\mathrm{CdS}$, thus resulting in the generation of new traps on the surface of the QDs, which led to the fluorescent quenching of QDs. It is has been proved that the copper ions can be highly selective and sensitive probed by using the luminescent QDs-MAO-mPEG based on the CdSe/CdS core/shell in chemical sensing analysis.

In addition, a typical liquid-phase quenching assay of the QDs-MAO-mPEG chemsensor with various concentration divalent copper ions is shown in Fig. 7. It can be seen that the fluorescence of QDs-MAO-mPEG become faint, and even disappeared in naked eyes with increasing $\left[\mathrm{Cu}^{2+}\right]$ above $1.0 \mu \mathrm{M}$. Compared with the quenching phenomenon of copper ions, no such remarkable effects from other metal ions at the $\mathrm{nM}$ scale on the fluorescence of QDs-MAO-mPEG have been observed, indicating that the QDs-MAO-mPEG sensor possesses excellent 

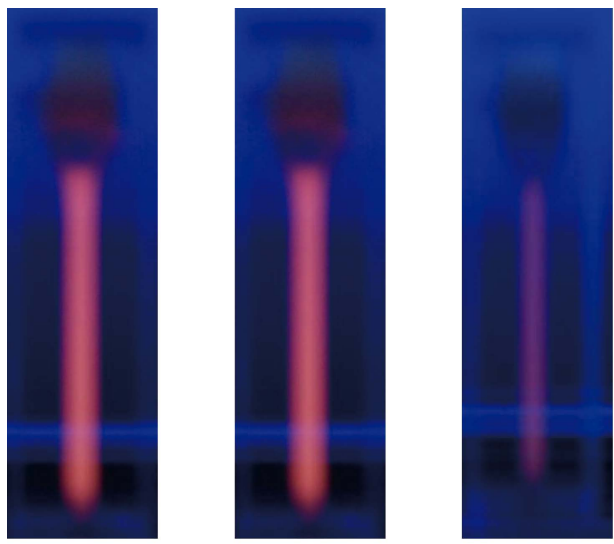

$0 \mu \mathrm{M}$
$0.5 \mu \mathrm{M}$

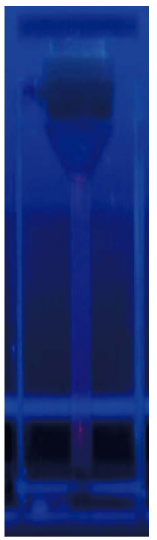

$2.5 \mu \mathrm{M}$

Fig. 7 Typical liquid-phase quenching assay of the QDs-MAO-mPEG sensor. Fluorescence emission spectra of QDs-MAO-mPEG $(1.0 \mu \mathrm{M}$, $298 \mathrm{~K}$, potassium dihydrogen phosphate and disodium phosphate ( $\mathrm{v}: \mathrm{v}=1: 1)$ buffer solution) in the presence of various concentrations of $\mathrm{Cu}^{2+}$ in $0,0.5,1.0$ and $2.5 \mu \mathrm{M}$. Fluorescence excitation was provided with a handheld low-wattage mineral lamp while set on "long wavelength" $(365 \mathrm{~nm})$. The image was acquired after 2 min of incubation.

selectivity and sensitivity to copper ions at a $\mu \mathrm{M}$ scale. It is thus concluded that the various binding affinity between QDs-MAO-mPEG and metal ions will help differentiate the binding of the ligand with its target copper ion from other metal ions, which will also provide a guideline for designing ion sensors in the future.

\section{Conclusion}

In the present work, a novel copper-sensitive fluorescent fluoroionophores was synthesized by modifying $\mathrm{CdSe} / \mathrm{CdS}$ core/shell QDs with MAO-mPEG, in which significant fluorescence quenching was observed upon binding with copper ions, indicating a high sensitivity and specificity of this chemosensor for copper ions. The presence of other metal ions had no significant interfering effects on the selectivity and sensitivity of $\mathrm{Cu}^{2+}$ and $\mathrm{Cu}^{+}$. It shows a potential application for multiplex sensing of different analytes through distinct ligand conjugation and functionalization of individual polymers combining with nano-materials, which provide useful information for design of fluoroionophores in the future.

\section{Acknowledgements}

This work was financially supported by projects of National Natural Science Foundation of China (20775010, 21075011, 21003014), Hunan Provincial Natural Science Foundation of China (09JJ3016, 10JJ5002), Foundation of Science and Technology Agency of Hunan Province of China (Nos. 2009WK4005, 2009FJ3173, 2010FJ3167), Foundation of Hunan

Provincial Key Laboratory of Materials Protection for Electric Power and Transportation of China (No. 2011CL05), and Program for New Century Excellent Talents in University (NCET-10-0138), Ministry of Education of the People's Republic of China.

\section{References}

1. M. Bruchez Jr., M. Moronne, P. Gin, S. Weiss, and A. P. Alivisatos, Science, 1998, 281, 2013.

2. W. C. Chan and S. Nie, Science, 1998, 281, 2016.

3. L. Qu and X. Peng, J. Am. Chem. Soc., 2002, 124, 2049.

4. B. Dubertret, P. Skourides, D. J. Norris, V. Noireaux, A. H. Brivanlou, and A. Libchaber, Science, 2002, 298, 1759.

5. X. Michalet, F. F. Pinaud, L. A. Bentolila, J. M. Tsay, S. Doose, J. J. Li, G. Sundaresan, A. M. Wu, S. S. Gambhir, and S. Weiss, Science, 2005, 307, 538.

6. A. J. Sutherland, Curr. Opin. Solid State Mater. Sci., 2002, $6,365$.

7. D. M. Willard, Anal. Bioanal. Chem., 2003, 376, 284.

8. C. J. Murphy and J. L. Coffer, Appl. Spectrosc., 2002, 56, $16 \mathrm{~A}$.

9. D. J. Crouch, P. O'Brien, M. A. Malik, P. J. Skabara, and S. P. Wright, Chem. Commun., 2003, 1454.

10. A. V. Isarov and J. Chrysochoos, Langmuir, 1997, 13, 3142.

11. E. Rabani, J. Chem. Phys., 2001, 115, 1493.

12. H. Mattoussi, J. M. Mauro, E. R. Golman, G. P. Anderson, V. C. Sundar, F. V. Mikulec, and M. G. Bawendi, J. Am. Chem. Soc., 2000, 122, 12142.

13. W. J. Jin, M. T. Fernández-Argüelles, J. M. CostaFernández, R. Pereiro, and A. Sanz-Medel, Chem. Commun., 2005, 883.

14. Y. Chen and Z. Rosenzweig, Anal. Chem., 2002, 74, 5132.

15. K. M. Gattás-Asfura and R. M. Leblanc, Chem. Commun., 2003, 2684.

16. H. Y. Xie, J. G. Liang, Z. L. Zhang, Y. Liu, Z. K. He, and D. W. Pang, Spectrochim. Acta, Part A, 2004, 60, 2527.

17. J. G. Liang, X. P. Ai, Z. K. He, and D. W. Pang, Analyst, 2004, 129, 619.

18. C. Bo and Z. Ping, Anal. Bioanal. Chem., 2005, 381, 986.

19. W. Guo, J. J. Li, Y. A. Wang, and X. Peng, J. Am. Chem. Soc., 2003, 125, 3901.

20. $\mathrm{Al}^{3+}, \mathrm{Mg}^{2+}, \mathrm{Ni}^{2+}, \mathrm{Pb}^{2+}$ and $\mathrm{Zn}^{2+}$ were used as their sulfate salts; $\mathrm{Co}^{2+}, \mathrm{Mn}^{2+}$ were used as their acetate salts; $\mathrm{Ag}^{+}$were used as their nitrate salts; while other metal ions were used as their chloride salts.

21. H. M. N. H. Irving, H. Freiser, and T. S. West, "IUPAC Compendium of Analytical Nomenclature, Definitive Rules", 1981, Pergamon Press, Oxford.

22. K. A. Connors, "Binding Constants, The Measurement of Molecular Complex Stability”, 1987, John Wiley \& Sons, New York, 339 - 343.

23. Z. Li, X. Zhu, C.-Q. Dong, X.-Y. Huang, H.-J. Chen, and J.-C. Ren, Chem. J. Chin. Univ., 2010, 31, 1905.

24. Y. F. Chen and Z. Rosenzweig, Anal. Chem., 2002, 74, 5132.

25. K. Youngjin, J. C. Robert, and H. T. Joseph, Nano Lett., 2001, $1,165$. 